\title{
Sentinel Lymph Node Biopsy for Breast Cancer: How Many Nodes are Enough?
}

\author{
SHAHEEN ZAKARIA, MBBS, ${ }^{1}$ AMY C. DEGNIM, MD, ${ }^{1}$ CELINA G. KLEER, MD, ${ }^{2}$ KATHLEEN A. DIEHL, MD, ${ }^{3}$ \\ VINCENT M. CIMMINO, MD, ${ }^{3}$ ALFRED E. CHANG, MD, ${ }^{3}$ LISA A. NEWMAN, MD, ${ }^{3}$ AND MICHAEL S. SABEL, $\mathrm{MD}^{3} *$ \\ ${ }^{1}$ Department of Surgery, Mayo Clinic, Rochester, Minnesota \\ ${ }^{2}$ Department of Pathology, University of Michigan Comprehensive Cancer Center, Ann Arbor, Michigan \\ ${ }^{3}$ Department of Surgery, University of Michigan Comprehensive Cancer Center, Ann Arbor, Michigan
}

\begin{abstract}
Introduction: Sentinel lymph node (SLN) biopsy using blue dye and radioisotope often results in the removal of multiple SLNs. We sought to determine whether there is a point where the surgeon can terminate the procedure without sacrificing accuracy. Methods: One thousand one hundred ninety-seven patients from University of Michigan and the Mayo Clinic undergoing SLN biopsy formed the study population. Surgeons removed all SLNs until counts within the axilla were less than $10 \%$ of the highest node ex vivo and recorded the order in which they were removed.

Results: The mean number of SLNs removed per patient was 2.5 (range 1-9). Approximately $42 \%$ of patients had three or more lymph nodes removed, while $19 \%$ had four or more lymph nodes removed. Eighteen percent of patients $(132 / 725)$ at University of Michigan and 22\% (103/472) at Mayo Clinic had a positive SLN. Ninety-eight percent (231/235) of patients with lymph node metastases were identified by the 3rd SLN while $100 \%$ were identified by the 4th SLN.

Conclusion: Among patients undergoing SLN biopsy for breast cancer, the only positive SLN is rarely identified in the 4th or higher node. Terminating the procedure at the 4th node may lower the cost of the procedure and reduce morbidity.

J. Surg. Oncol. 2007;96:554-559. ( 2007 Wiley-Liss, Inc.
\end{abstract}

KEY WoRDs: breast cancer; sentinel lymph; node biopsy; lymph nodes

\section{INTRODUCTION}

Sentinel lymph node (SLN) biopsy has become a standard method for staging the axilla in clinically node negative patients with breast cancer [1,2]. The benefit of SLN biopsy in providing accurate staging information while avoiding the morbidity of a complete axillary lymph node dissection has been well-documented [3-5]. The SLN is defined as the first lymph node to receive lymphatic drainage from the tumor and is therefore the most likely lymph node to harbor micrometastases if they exist. The practical definition of the SLN is different, however. With the combined use of technetium $99 \mathrm{~m}$-sulfur colloid $\left({ }^{99 \mathrm{~m}} \mathrm{Tc}\right)$ and blue dye to identify SLN, multiple lymph nodes are often found containing tracer. Any lymph node that exhibits radioactivity, is blue or has a blue afferent lymphatic channel, or is palpably suspicious is excised and labeled as a SLN [6].

A dual tracer technique with blue dye and radioisotope increases both the success rate and the accuracy of the procedure [7-9], but also increases the number of lymph nodes removed per case $[10,11]$. The degree of radioactivity necessary to excise and define a lymph node as 'sentinel' varies among surgeons [6,12-14]. Several authors have demonstrated that the hottest SLN (the node with the highest counts) is not always the node to harbor metastasis [10,15-17], and so a threshold for excising additional SLNs is necessary. What that threshold should be, however, remains in question. With a lower threshold, the surgeon is less likely to miss a positive SLN but more likely to remove more lymph nodes per case. Furthermore, the absolute number of counts in the node varies depending on factors such as the dose and

*Correspondence to: Michael S. Sabel, MD, 3304 Cancer Center, 1500 East Medical Center Drive, Ann Arbor, MI 48109-0932; Fax: 734-647-9647.

E-mail: msabel@umich.edu

Received 4 December 2006; Accepted 27 June 2007

DOI 10.1002/jso.20878

Published online 8 August 2007 in Wiley InterScience (www.interscience.wiley.com). 
type of radioactive colloid, time interval from injection to operation, the type of gamma probe, and its calibration. The purpose of this analysis was to determine whether there is a threshold (based on number of SLNs removed) after which the surgeon does not need to excise additional lymph nodes.

\section{MATERIALS AND METHODS}

Medical records were reviewed for 1,197 breast cancer patients who underwent successful lymphatic mapping and SLN biopsy between October 1998 and December 2004 at the University of Michigan $(n=725)$ and from March 2003 to December 2005 at the Mayo Clinic $(n=472)$. Approval of this study was granted by the respective Institutional Review Boards. All patients underwent injection of ${ }^{99 \mathrm{~m}} \mathrm{Tc}$-sulfur colloid (CIS-US, Inc., Bedford, MA) either peri-tumorally or periareolar; injection was either the night before or the morning of the procedure. Isosulfan blue dye (Lymphazurin 1\%; Hirsch Industries, Inc., Richmond, VA) was utilized in 710 patients (except 19 patients who had the procedure performed during a national shortage) at the University of Michigan, while methylene blue dye (American Regent, Inc., Shirley, NY) was used in all the patients at the Mayo Clinic. Blue dye was injected either peri-tumorally or at the subareolar location, followed by 3-5 min of massage to promote lymphatic flow. A handheld gamma probe (Navigator GPS; US Surgical) was used to identify transcutaneous hot spots and to guide dissection of the $\operatorname{SLN}(\mathrm{s})$.

Lymph nodes were removed and labeled as SLNs if they were palpably suspicious, blue, had blue-stained afferent lymphatic vessels, or were hot, defined as the hottest node plus any lymph node with at least $10 \%$ of the ex vivo counts per minute (CPM) of the hottest node. The SLNs were numbered in the order they were excised, so the first node was not necessarily the hottest node based on CPM. At the Mayo Clinic, a preliminary frozen section was performed on all SLNs, while this was performed selectively at the University of Michigan. If the SLN was negative for tumor, the node was measured and cut entirely along its longitudinal axis into sections of $1.5-2 \mathrm{~mm}$ thickness. The sections were submitted in formalin for paraffin section histology. At least four additional levels were examined with hematoxylin and eosin (H\&E) and cytokeratin antibody (AE1/3, monoclonal antibody, 1/250; Boehringer Mannheim, Indianapolis, IN), together with a negative control. The routine use of IHC for cytokeratin was discontinued at the University of Michigan in July 2002. For the Michigan dataset, sentinel nodes positive by IHC but negative by $\mathrm{H} \& \mathrm{E}$ were considered negative. In contrast, IHC was utilized uniformly for all Mayo Clinic patients. Of 472 patients in the Mayo dataset, information on IHC staining was recorded for 414 patients. Of these, five patients had positive SLNs based on IHC alone (all were $\mathrm{NOi+}$ ). IHC was not used on any non-SLNs.

\section{RESULTS}

The study group consisted of 1,197 patients; 725 from the University of Michigan and 472 from the Mayo Clinic. The median age of the patients was 55 years (range, 17-85 years) in the Michigan dataset and was 64 years (range, 24-90 years) in the Mayo dataset. Overall, $71 \%$ of patients had invasive ductal carcinoma and $11 \%$ had invasive lobular carcinoma. The patient and tumor characteristics presented in Table I were similar between the two institutions.

The mean number of SLNs removed by the surgeon in both the Michigan (range, 1-9) as well as in the Mayo dataset (range, 1-8) was 2.5. The number of lymph nodes surgically removed and pathologically examined at each institution is shown in Table II. Often, the surgeon removes and labels a single node as a SLN, but the pathologist identifies more than one node within the specimen. Based on pathology reports, the mean number of SLNs examined per patient was 3.1 (range, 1-24) at the University of Michigan and 2.8 (range, 1-9) at the Mayo Clinic. The frequency distribution of the number of SLNs removed by the surgeon and identified by the

TABLE I. Patient and Tumor Characteristics

\begin{tabular}{|c|c|c|c|c|}
\hline & \multicolumn{2}{|c|}{$\begin{array}{l}\text { Michigan dataset, } \\
n=725\end{array}$} & \multicolumn{2}{|c|}{$\begin{array}{c}\text { Mayo dataset, } \\
\mathrm{n}=472\end{array}$} \\
\hline & $\mathrm{n}$ & $\%$ & $\mathrm{n}$ & $\%$ \\
\hline \multicolumn{5}{|l|}{ Tumor histology } \\
\hline Invasive ductal & 511 & 70 & 343 & 73 \\
\hline Invasive lobular & 68 & 10 & 55 & 12 \\
\hline DCIS & 55 & 7 & 42 & 9 \\
\hline DCIS with microinvasion & 35 & 5 & - & - \\
\hline Other & 56 & 8 & 32 & 6 \\
\hline \multicolumn{5}{|l|}{ Tumor size } \\
\hline Tis & 55 & 8 & 42 & 9 \\
\hline $\mathrm{T} 1 \mathrm{mic}$ & 35 & 5 & 8 & 2 \\
\hline T1a & 76 & 10.5 & 25 & 5 \\
\hline $\mathrm{T} 1 \mathrm{~b}$ & 181 & 25 & 91 & 20 \\
\hline $\mathrm{T} 1 \mathrm{c}$ & 249 & 35 & 193 & 42 \\
\hline $\mathrm{T} 2$ & 111 & 15.5 & 86 & 18 \\
\hline $\mathrm{T} 3$ & 7 & 1 & 18 & 4 \\
\hline Unknown & 11 & & 9 & \\
\hline \multicolumn{5}{|l|}{ Tumor grade (invasive) } \\
\hline 1 & 144 & 21.3 & 104 & 25 \\
\hline 2 & 283 & 42.4 & 226 & 53 \\
\hline 3 & 127 & 19.1 & 87 & 22 \\
\hline Unknown & 116 & 17.2 & 13 & \\
\hline \multicolumn{5}{|l|}{ Estrogen receptor (invasive) } \\
\hline Positive & 448 & 66.8 & 374 & 87 \\
\hline Negative & 159 & 23.6 & 56 & 13 \\
\hline Unknown & 63 & & - & \\
\hline
\end{tabular}


TABLE II. Number of SLNs Excised and Examined

\begin{tabular}{|c|c|c|c|c|c|c|c|c|}
\hline \multirow[b]{3}{*}{ No. of SLN/s } & \multicolumn{4}{|c|}{ Excised by surgeon } & \multicolumn{4}{|c|}{ Examined by pathologist } \\
\hline & \multicolumn{2}{|c|}{ Michigan dataset } & \multicolumn{2}{|c|}{ Mayo dataset } & \multicolumn{2}{|c|}{ Michigan dataset } & \multicolumn{2}{|c|}{ Mayo dataset } \\
\hline & $\mathrm{n}$ & $\%$ & $\mathrm{n}$ & $\%$ & $\mathrm{n}$ & $\%$ & $\mathrm{n}$ & $\%$ \\
\hline 1 & 168 & 23 & 97 & 22 & 147 & 20 & 91 & 19 \\
\hline 2 & 261 & 36 & 151 & 34.5 & 216 & 30 & 133 & 28 \\
\hline 3 & 162 & 22 & 99 & 23 & 156 & 22 & 115 & 24 \\
\hline 4 & 81 & 11 & 51 & 12 & 77 & 11 & 67 & 14 \\
\hline 5 & 30 & 4 & 21 & 5 & 44 & 6 & 31 & 6.5 \\
\hline 6 & 12 & 1.7 & 10 & 2 & 28 & 4 & 21 & 4 \\
\hline 7 & 6 & 0.8 & 1 & 0.2 & 11 & 1.5 & 9 & 2 \\
\hline 8 & 2 & 0.3 & 2 & 0.4 & 15 & 2 & 3 & 0.6 \\
\hline 9 & 2 & 0.3 & - & - & 8 & 1 & 2 & 0.4 \\
\hline 10 & - & - & - & - & 9 & 1 & - & - \\
\hline 11 & - & - & - & - & 6 & 0.8 & - & - \\
\hline$\geq 12$ & - & - & - & - & 5 & 0.7 & - & - \\
\hline
\end{tabular}

pathologist was remarkably similar between the two institutions. Overall, $42 \%$ of patients had three or more SLNs removed while $19 \%$ had four or more lymph nodes removed. Nearly half of the patients had three or more and a quarter had four or more nodes examined histologically.

The position of the first positive node in the order of SLN removal is presented in Table III. Of the 725 patients undergoing successful SLN biopsy at the University of Michigan, 132 (18\%) had metastasis to at least one SLN. There was an average of 1.5 positive SLN per nodepositive patient. Of the 132 patients with lymph node metastases, $66 \%$ (87 patients) were positive in the first lymph node removed by the surgeon, $86 \%$ (114 patients) by the second node, $98 \%$ (130) by the third node, and $100 \%$ ( 132 patients) by the 4 th node. There were no cases where the surgeon needed to excise more than four nodes to identify metastases.

Of the 472 patients having successful SLN biopsy at the Mayo Clinic, 103 (22\%) had metastasis to at least one SLN (Table III). Of the 103 patients with SLN metastases, 87\% (90) were positive in the 1st node removed by the surgeon, $95 \%$ (98) by the 2nd node, $98 \%$ (101) by the 3rd node, and $100 \%$ (103) by the 4 th node. Similar to the Michigan study population, there were no cases where the surgeon needed to excise more than four nodes to identify metastases. Despite the use of IHC at the Mayo Clinic and the five additional patients who were positive by IHC only, the routine use of IHC did not alter these results.

Terminating the procedure after the 3rd node would have shortened the operation for $133(18 \%)$ at Michigan and $111(26 \%)$ patients at Mayo, and avoided histological evaluation of 223 and 137 nodes, respectively, but would have missed disease in 4 of 235 patients $(1.7 \%)$. In the Michigan dataset, terminating the procedure after the after the 4th node removed would not have missed any positive cases but overall spared 52 patients $(7 \%)$ the extra time in the OR to identify additional nodes and the histologic evaluation of 90 additional nodes. In the Mayo dataset, this approach would spare 34 patients from the additional time in the OR ( $7 \%$ of the total) and the histologic evaluation of 52 nodes without missing any positive SLNs (Table IV).

\section{DISCUSSION}

Sentinel lymph node biopsy has evolved as the preferred technique for axillary staging in breast cancer.

TABLE III. Order In Which the First Positive SLN was Removed

\begin{tabular}{|c|c|c|c|c|c|c|}
\hline & \multicolumn{3}{|c|}{ Michigan dataset } & \multicolumn{3}{|c|}{ Mayo dataset } \\
\hline & $\mathrm{n}$ & $\%$ Of cases & $\begin{array}{c}\text { Cumulative } \\
\text { frequency (\%) }\end{array}$ & $\mathrm{n}$ & $\%$ of Cases & $\begin{array}{c}\text { Cumulative } \\
\text { frequency }(\%)\end{array}$ \\
\hline Node negative & 593 & 82 & - & 369 & 78 & - \\
\hline Node positive & 132 & 18 & - & 103 & 22 & - \\
\hline 1st Node removed & 87 & 66 & 66 & 90 & 87 & 87 \\
\hline 2nd Node removed & 27 & 20 & 86 & 8 & 8 & 95 \\
\hline 3rd Node removed & 16 & 12 & 98 & 3 & 3 & 98 \\
\hline 4th Node removed & 2 & 2 & 100 & 2 & 2 & 100 \\
\hline
\end{tabular}


TABLE IV. Number of Excess SLNs Removed by the Surgeon Beyond the 4th SLN

\begin{tabular}{lccccc}
\hline & \multicolumn{2}{c}{ Michigan dataset } & & \multicolumn{2}{c}{ Mayo dataset } \\
\cline { 2 - 3 } \cline { 5 - 6 } No. of excess SLNs & No. of patients & $\begin{array}{c}\text { No. of excess } \\
\text { nodes removed }\end{array}$ & & No. of patients & $\begin{array}{c}\text { No. of excess } \\
\text { nodes removed }\end{array}$ \\
\hline 1 & 30 & 30 & & 21 & 21 \\
2 & 12 & 24 & & 10 & 3 \\
3 & 6 & 18 & & 2 & 8 \\
4 & 2 & 10 & & - & 52 \\
5 & $52(7 \%$ of total $)$ & 90 & & $34(7 \%$ of total $)$ & \\
Total & & & &
\end{tabular}

Studies have shown that the SLN can be identified in $96 \%$ of cases with a low false negative rate [18-20]. The mean number of SLNs removed in the SLN biopsy procedure ranges from 1.2 to 3.4 per patient, with a total number ranging from 1 to 8 in the published literature $[11,13,17,18]$. Removal of more SLNs increases time in the OR, time for the pathologist, costs of the procedure, and axillary morbidity [21]. The ACOSOG Z0010 prospective trial of SLN biopsy in breast cancer reported significantly higher rates of axillary seroma and wound infection in patients who had five or more SLNs removed, compared to those with up to four nodes removed [21]. It is therefore worthwhile to determine how many SLNs should be excised to adequately stage the axilla. In this study we approached this question with a simple criterion - the number of SLNs removed by the surgeon. We sought to discover whether there is a numerical threshold after which the surgeon does not need to excise additional lymph nodes without sacrificing accuracy.

With excision of all blue, palpably suspicious, and hot nodes based upon a $10 \%$ rule, $19 \%$ of patients in our study had four or more lymph nodes removed, and $28 \%$ had a similar number of SLNs examined pathologically. Ninety-eight percent of SLN positive patients had metastasis in the first three SLNs removed, with the remaining 2\% found in the fourth SLN. There were no cases where the surgeon needed to excise more than four SLNs to identify SLN metastases. These data suggest that it is rare to identify nodal metastasis in the 4th or higher sentinel node, assuming that all nodes meeting the definition of a SLN (see Material and Methods) have been removed.

The optimal techniques for SLN biopsy have been debated, but there is considerable support for a dual tracer technique [7-9], using both blue dye and radioisotope injection. Although a single SLN should in theory accurately reflect the status of the nodal basin, published clinical trial results confirm that in practice the false negative rate is lower with excision of more than one SLN [22]. When radioisotope is used for lymphatic mapping, some threshold of radioactivity is selected to define the number of SLNs removed. Various count ratios have been used: SLN to background, SLN to non-SLN, or SLN to hottest SLN. Most surgeons use a ratio of the in vivo CPM to the background radioactivity or to the ex vivo node counts compared to that of the node with the highest CPM $[6,10,12-15,23]$. A common practice is to remove all nodes with counts greater than $10 \%$ of the hottest node, based on results from published investigations $[10,23]$.

Several groups have examined this subject and found similar results, with all positive nodes identified by the 4th node (Table V), although there were a few exceptions. The Axillary Lymphatic Mapping Against Nodal Axillary Clearance (ALMANAC) Trialists group reported that $99.6 \%$ of node positive patients were identified within the first four sentinel nodes removed [23]. McCarter et al. [24] from Memorial Sloan Kettering

TABLE V. Studies Examining How Many Nodes Needed to be Removed before Identifying All Positive SLN

\begin{tabular}{|c|c|c|c|c|c|c|c|}
\hline Author and institution & \# Pos SLN & 1st Node (\%) & 2nd Node $(\%)$ & 3rd Node $(\%)$ & 4th Node (\%) & 5 th Node $(\%)$ & 6 th-8th Node $(\%)$ \\
\hline MDACC/Duke [29] & $278(11 \%)$ & 79 & 96 & 99 & 100 & N/A & N/A \\
\hline University of Michigan & $132(18 \%)$ & 66 & 86 & 98 & 100 & N/A & N/A \\
\hline Mayo Clinic & $103(22 \%)$ & 87 & 95 & 98 & 100 & N/A & N/A \\
\hline Beaumont Hospital [25] & $172(24 \%)$ & 76.6 & 91 & 97 & 98.8 & 99.4 & 100 \\
\hline Ludwig Boltzmann [30] & $105(40 \%)$ & 91.4 & 99 & 100 & N/A & N/A & N/A \\
\hline Virginia Mason [31] & $129(24 \%)$ & 65 & 98.4 & 99.2 & 100 & N/A & N/A \\
\hline MSKCC [24] & $241(15 \%)$ & 75.3 & 92.9 & 98 & 99.1 & 99.6 & 100 \\
\hline Wagga Wagga [32] & $33(29 \%)$ & 88 & 97 & 100 & N/A & N/A & N/A \\
\hline University South Florida [33] & $128(27 \%)$ & 89 & 98.4 & 99.2 & 100 & N/A & N/A \\
\hline Ohio State University [34] & $104(29 \%)$ & 83 & 97 & 98 & 99 & 100 & N/A \\
\hline
\end{tabular}


Cancer Center, reported that $99.1 \%$ of patients with a positive SLN were found by the fourth SLN removed, and $99.6 \%$ were found in the first five nodes removed. Woznick et al. [25] from Beaumont hospital evaluated 172 patients with positive SLN and found that $100 \%$ of positive nodes were found by six nodes removed. Chagpar et al. [26] reviewed data from the Louisvile Breast Sentinel Lymph Node Study and found that $100 \%$ of disease was not identified until $>10$ nodes were removed, although after 4 nodes were removed, $98.5 \%$ of positive SLN were identified. Even in these reports where four nodes did not identify $100 \%$ of disease, very few patients $(<2 \%)$ would have been adversely affected by terminating the procedure at the 4th node. Mathematical models of the impact of a false-negative SLN biopsy on 10 -year breast cancer mortality demonstrate an almost negligible impact with $1-2 \%$ changes in the false negative rate [27].

Sentinel lymph node biopsy has an inherent false negative rate in the range of $4-10 \%$ [18-20]. Based upon the findings of this study and the others summarized above, terminating the SLN biopsy procedure at four nodes should identify a positive SLN in almost all cases and thus increase the false negative rate by less than $1 \%$. Attempts have been made by various researchers to define optimal surgeon experience for reducing false negative rate associated with SLN biopsy. Anywhere between 5 [18] and 20 procedures have been used as the cut-off point [28]. However, most of the validation studies for SLN biopsy technique are from the late 1990s and did not take surgeon's experience into account. Data from pre-randomization surgical training for the NSABP B-32 trial suggests that adherence to a standardized protocol of SLN biopsy procedure can lead to technical success rates of $96 \%$ among surgeons with a wide range of expertise for this procedure [18]. Based on these points it seems reasonable to presume that terminating the SLN biopsy procedure at four nodes should have minimal incremental impact on the false negative rate of SLN biopsy procedure, as long as it is performed after completion of recommended training in the procedure.

Some surgeons do advocate continuing the SLN biopsy procedure until all hot nodes are removed. However, there is a cost to this approach. Although it may seem callous to examine the costs of breast cancer care, the cost of health care in the United States is a significant problem. Even though the number of excess SLNs removed and examined per patient is small, given the additional time spent in the OR and time for the pathologist to thin-section each node, the cumulative effect of this practice on health care resources is substantial. A challenge to surgeons is to reduce unnecessary costs without compromising patient care; thus, physicians should bear in mind the costs of new technologies and treatments in cancer care. While it is desirable to pursue the highest level of accuracy with the SLN biopsy procedure, the relative gains in accuracy from excision of multiple nodes may not be justified by the attendant increased cost and morbidity. In a procedure with a known false negative rate of approximately $7 \%$, removing more than four SLNs will contribute little to improved accuracy, based on the available data.

In summary, our findings and other published literature support the conclusion that a positive sentinel node will be identified in $100 \%$ of cases within the first four sentinel nodes removed by the surgeon, according to current practices. Terminating the SLN biopsy procedure at four lymph nodes will have no impact on the false negative rate, but there is a cost savings associated with setting such a threshold. As long as the surgeon is confident that the blue and hottest node(s) have been removed, it is reasonable to limit sentinel node removal to four nodes in order to reduce both the cost as well as the morbidity of the procedure.

\section{REFERENCES}

1. Veronesi U, Paganelli G, Viale G, et al.: A randomized comparison of sentinel node biopsy with routine axillary dissection in breast cancer. N Engl J Med 2003;349:546-553.

2. Giuliano AE, Dale PS, Turner RR, et al.: Improved staging of breast cancer with sentinel lymphadenectomy. Ann Surg 1995;3: 394-401

3. Blanchard DK, Donohue JH, Reynolds C, et al.: Relapse and morbidity in patients undergoing sentinel lymph node biopsy alone or with axillary dissection for breast cancer. Arch Surg 2003; 138:482-488.

4. Ivens D, Hoe AL, Podd TJ, et al.: Assessment of morbidity from complete axillary dissection. Br J Cancer 1992;66:136-138.

5. Sener SF, Winchester DJ, Martz CH: Lymphedema after sentinel lymphadenectomy for breast carcinoma. Cancer 2001;92:748752.

6. Morton DL, Bostick PJ: Will the true sentinel node please stand? Ann Surg Oncol 1999;6:12-14.

7. Degnim AC, Oh K, Cimmino VM, et al.: Is blue dye indicated for sentinel lymph node biopsy in breast cancer patients with a positive lymphoscintigram? Ann Surg Oncol 2005;12:712 717.

8. McMasters KM, Tuttle TM, Carlson DJ, et al.: Sentinel lymph node biopsy for breast cancer: A suitable alternative to routine axillary dissection in multi-institutional practice when optimal technique is used. J Clin Oncol 2000;18:2560-2566.

9. Cody HS, Fey J, Akhurst T, et al.: Complementarity of blue dye and isotope in sentinel node localization for breast cancer: Univariate and multivariate analysis of 966 procedures. Ann Surg Oncol 2001:8:13-19.

10. Martin RCG, Edwards MJ, Wong SL, et al.: Practical guidelines for optimal gamma probe detection of sentinel lymph nodes in breast cancer: Results of a multi-institutional study. Surgery 2000; 128:139-144.

11. Borgstein PJ, Pijpers R, Comans EF, et al.: Sentinel lymph node biopsy in breast cancer: Guidelines and pitfalls of lymphoscintigraphy and gamma probe detection. J Am Coll Surg 1998;186: 275-283.

12. Albertini JJ, Lyman GH, Cox C, et al.: Lymphatic mapping and sentinel node biopsy in the patient with breast cancer. JAMA 1996;276:1818-1822.

13. Krag D, Weaver D, Ashikaga T, et al.: The sentinel node in breast cancer: A multicenter validation study. N Engl J Med 1998;339: 941-946. 
14. Nathanson SD: Will the true sentinel node please stand? (Letter). Ann Surg Oncol 1999;6:514-516.

15. Camp ER, Cendan JC, Feezor R, et al.: The hottest sentinel lymph node is not always the positive node. Am Surg 2004;70:475-478.

16. Bourgeois P, Nogaret JM, Veys I, et al.: How "hot" is the pathologically positive sentinel lymph node in breast cancer patients? Nucl Med Commun 2003;24:513-518.

17. Tafra L, Lannin DR, Swanson MS, et al.: Multicenter trial of sentinel node biopsy for breast cancer using both technetium sulfur colloid and isosulfan blue dye. Ann Surg 2001;233:51-59.

18. Harlow SP, Krag DN, Julian TB, et al.: Prerandomization surgical training for the NSABP-B32 trial-a randomization phase III clinical trial to compare sentinel node resection to conventional axillary dissection in clinically node negative breast cancer. Ann Surg 2005;241:48-54.

19. Veronesi U, Paganelli G, Galimberti V, et al.: Sentinel node biopsy to avoid axillary dissection in breast cancer with clinically negative lymph nodes. Lancet 1997;349:1864-1867.

20. Giuliano AE, Jones RC, Brennan M, et al.: Sentinel lymphadenectomy in breast cancer. J Clin Oncol 1997;15:2345-2350.

21. Wilke LG, McCall LM, Posther KE, et al.: Surgical complications associated with sentinel lymph node biopsy: Results from a prospective international cooperative group trial. Ann Surg Oncol 2006; $13: 491-500$.

22. Wong SL, Edwards MJ, Chao C, et al.: Sentinel lymph node biopsy for breast cancer; impact of the number of sentinel nodes removed on the false-negative rate. J Am Coll Surg 2001;192: 684-689.

23. Goyal A, Newcombe RG, Chhabra A, et al.: Factors affecting failed localization and false-negative rates of sentinel node biopsy in breast cancer-results of the ALMANAC validation phase. Breast Cancer Res Treat 2006;99:203-208.

24. McCarter MD, Yeung H, Fey J, et al.: The breast cancer patient with multiple sentinel nodes: When to stop? J Am Coll Surg 2001; 192:692-697.
25. Woznick A, Franco M, Bendick P, Benitez PR: Sentinel lymph node dissection for breast cancer: How many nodes are enough and which technique is optimal? Am J Surg 2006;191:330333.

26. Chagpar AB, Scoggins CR, Martin RCG II, et al.: Are 3 sentinel nodes sufficient? Arch Surg 2007;142:456-460.

27. Vaidya JS, Dewar JA, Brown DC, et al.: A mathematical model for the effect of a false-negative sentinel node biopsy on breast cancer mortality: A tool for everyday use. Breast Cancer Res 2005;7:225-227.

28. McMasters KM, Wong SL, Chao C, et al.: Defining the optimal surgeon experience for breast cancer sentinel lymph node biopsy: A model for implementation of new surgical techniques. Ann Surg 2001;234:292-300.

29. Grubbs E, Bennett K, Westlund R, et al.: Sentinel lymph node biopsy in breast cancer: How many nodes need to be removed (abstract). Ann Surg Oncol 2007;14 41.

30. Schrenk P, Rehberger W, Shamiyeh A, et al.: Sentinel node biopsy for breast cancer: Does the number of sentinel nodes removed have an impact on the accuracy of finding a positive node? J Surg Oncol 2002;80:130-136.

31. Duncan M, Cech A, Wechter D, et al.: Criterial for establishing the adequacy of a sentinel lymphadenectomy. Am J Surg 2004; 187:639-642.

32. Low KS, Littlejohn DR: Optimal number of sentinel nodes after intradermal injection isotope and blue dye. ANZ J Surg 2006;76: $472-475$.

33. Zervos EE, Badgwell BD, Abdessalam SF, et al.: Selective analysis of the sentinel node in breast cancer. Am J Surg 2001; 182:372-376

34. Povoski SP, Young DC, Walker MJ, et al.: Re-emphasizing the concept of adequacy of intraoperative assessment of the axillary sentinel lymph nodes for identifying nodal positivity during breast cancer surgery. World J Surg Oncol 2007;5:18 